\title{
ANALISE BOTÂNICA DE UM CAMPO DE PASTAGEM NO ESTADO DO RIO DE JANEIRO
}

\author{
A. L. PEIXOTO**, S. M. CARVALHO***, e M. M. T. DA ROSA
}

Docentes do Departamento de Biologia Ve getal do Instituto de Biologia da Universi dade Federal Rural do Rio de Janeiro, 23.460 Itaguaí, RJ.

** Bolsis ta do CNPq.

*** Bolsista da CAPES.

\section{RESUMO}

O presente estudo trata da análise fitossociológica de uma área de pastagem localizada no Município de Vassouras, Estado do Rio de Janeiro, e é parte de um projeto que vem sendo desenvolvido por docentes do Instituto de Biologia da UFRRJ sobre Biologia e Ecologia de Hemípteros do gênero Dysdercus.

$\mathrm{Na}$ área foram estabelecidos quadrados de $1 \mathrm{~m}^{2}$, dos quais se obtiveram dados de freqüência, densidade e dominância. A somatória dos valores relativos destes dados fornece o IVI (indice de Valor de Importância). As gramíneas que formam a pastagem quando somadas atingem 114,90 IVI. Todas as gramíneas atingem 161,18 IVI. As espécies de gramíneas que são biologicamente infestantes do pasto podem ser consideradas como forrageiras associadas. Wedelia paludosa DC. é a espécie de dicotiledonea de mais alto IVI $=22,65$. Esta espécie, nos meses de elevadas pluviosidade e temperatura, associadas a um pastoreio intenso das forrageiras, desenvolve-se cobrindo parcialmente $o$ pasto.

Embora na área tenham sido encontradas 27 famílias com 67 espécies, apenas 37 estão representadas nos quadrados, as outras podendo ser consideradas esporádicas na comunidade.

PALAVRAS CHAVE: Fitossociologia, pas tagem e invasoras.

\section{SUMMARY}

BOTANICAL ANALYSIS OF A PASTURE FIELD IN THE STATE OF RIO DE JANEIRO

A pasture field in Vassouras, State of Rio de Janeiro, was analysed from the viewpoint of plant sociology. This study is partt of a project being developed by several professors from the Institute of Biology of UFRRJ on the Biology and Ecology of Hemiptera of the genus Dysdercus.

Quadrats of one square meter were located in the field and for each one, data of frequency, density and dominance were collected. The sum of the relative value of this data produces the IVI (Importance Value Index). The grasses making up the pasture added up to 114,9 IVI. All grasses summed up gave an IVI of 161,18 , grass species infesting the pasture were considered associated forage plants. Wedelia paludosa DC. is the species of Dicotyledoneae with the highest IVI $=22,65$. During the high temperatures and rainfall months, associated with intense grazing of the forage plants, this species can develop until it partially covers the field.

Although 27 families and 67 species were counted in the pasture field, only 37 are represented in the quadrats; the others were considered sporadic in the community.

KEYWORDS: Pasture, weeds, plant socio logy.

\section{INTRODUC̣ÃO}

A análise fitossociológica tem sido valioso subsídio para o estudo do comportamento de ecossistemas naturais. Embora estes estudos sejam imprescindiveis para uma melhor compreensão do comportamento de comunidades artificiais, eles são ainda escassos. Informações fitossociológicas qualitativas e quantitativas de populações vegetais, vêm sendo utilizadas recentemente na interpretação da dinâmica de escossistemas artificiais como também no estudo de plantas invasoras de culturas (10).

Numa área de pastagem no Municipio de Vassouras, RJ, foi feito o levantamento florístico e a análise fitossociológica como base para a interpretação da dinâmica vegetacional. Este estudo, aliado a muitos outros que vêm sendo desenvolvidos, fornecerão dados para melhor entendimento da biologia e ecologia de Hemípteros do gênero Dysdercus (2, 3, 14 ). 


\section{MATERIAIS E MÉTODOS}

O trabalho foi realizado numa área de pastagem que faz parte da Fazenda Oriente, localizada no Municipio de Vassouras, Estado do Rio de Jan eiro, a 27 $\mathrm{Km}$ da Universidade Federal Rural do Rio de Janeiro, tendo aces so no $\mathrm{Km} 18$ da Estrada RJ 125.

A área em estudo compreende parte de um pasto com cerca de $6.200 \mathrm{~m}^{2}$. O solo é de textura média, argilo-arenoso e ácido. A distribuição de nutrientes não é uniforme, havendo carência nas elevações e excesso nas planícies. Apresenta-se parcialmente inundada nos meses de intensa pluviosidade (dezembro a abril) devido ao aumento do nível do cór rego que a corta.

O levantamento floristico foi realizado coletando-se material florífero e frutífero em um período anual ( julho de 1978 a agosto de 1979) em excursões quinzenais, anotando-se dados de periodicidade, sociabilidade e vitalidade. O material coletado sofreu o método normal de secagem e prensagem, encontrando-se depositado no Herbário da Universidade Fed eral Rural do Rio de Janeiro (RBR ). A identificação botânica das espécies foi feita através de chaves analisticas, monografias de famílias e gêneros e comparação com exsicatas dos Herbários RBR e do Jardim Botânico do Rio de Janeiro (R).

A análise sociológica inicial foi feita pelo método de estimativa de abundância e densidade de Braun-Blanquet (5) . Este método vem sendo largamente empregado para análise de plantas daninhas em arrozais (15), em cafe zais (10), em cana de açúcar (11) e em pastagen s artificiais (12).

Também foram marcados e analisa dos 12 quadrados de $1 \mathrm{~m}^{2}$ durante o mês de março. Coletaram-se dados de densidade, freqüência e cobertura. As discussões são feitas com base no indice de Valor de Importância (IVI). Este indice foi introduzido por Curtis \& McIntosh (8) para vegetação arbórea em Wisconsin (U.S.A.), usado por Cain et al. (6) também em vegetação arbórea no Estado do
Pará e por Araújo \& Peixoto (4) em vegetação herbáceo-arbustiva no Rio de Janeiro, com resultados satisfatórios.

\section{RESULTADOS E DISCUSSÃO}

Os campos de pastagens da Fazenda Oriente são formados em área primitivamente cobertos de matas mais ou menos densas nas regiões elevadas e por planícies pantanosas nas baixadas. Esta região é cortada pelo Rio Santana, que originalmente tinha leito raso e por ocasião das chuvas inundava a área. Â partir de 1944 a região começou a ser modificada com desmatamentos sucessivos, dragagem do rio e formação de pastos. Da vegetação primitiva restam apenas núcleos esparsamente distribuidos e árvores remanescentes.

A cobertura vegetal dos campos é herbácea, uniforme, com dominância de gramíneas, possuindo quatro espécies cultivadas : capim-colonião (Panicum maxi-

mum Jacq. ), capim-elefante (Pennisetum purpureum Schum. ), capim-marmelada (Brachiaria plantaginea (Link.) Hitch.) e capimangola (Brachiaria purpurascens Henr. ), que foram plantadas inicialmente por sementes, sendo que no campo estudado não se encontrou o capim-colonião.

Consideraram-se os indivíduos presentes na área em estudo como constituintes de uma fitocenose que na classificação de Alechin (1) representaria uma comunidade de grupamento instável devido a um fator biótico persistente (pastoreio ).

Aos campos de pastagens adicionamse expontaneamente uma certa quantidade de espécies formando uma comunidade vegetal com características que dependem basicamente das condições de solo, do regime hídrico e do pastoreio. Nesta vegetação há espécies que podem ser consideradas inócuas, pois, são aceitas pelo gado e associam-se com facilidade às gramineas forrageiras. Algumas são indesejáveis por sufocarem as forrageiras ou por terem propriedades tóxicas (12) ; outras são benéficas pelo alto valor nutri tivo e adubação verde do solo.

No levantamento floristico foram 
encontradas 27 famílias com 67 espécies (incluindo as gramineas cultivadas) que são citadas por ordem alfabética de família no Quadro 1. As famílias com maior número de espécies são Gramineae com 10 espécies, Compositae e Leguminosae com 7 espécies cada uma.

Nos quadrados foram encontradas 19 famílias com 37 espécies. Em pastagens na Estação Experimental de També, em Pernambuco, Sarmento (17) .encontrou 12 famílias sendo as mais bem representadas Gramineae com 22 espécies e Leguminosae com 9 espécies. Ferreira

Laca-Buendia (9), em levantamento de espécies consideradas infestantes em áreas cultivadas no Estado de Minas Gerais, citam 29 famílias com 295 espécies, sendo as familias mais expressivas Compositae com 61, Gramineae com 43 e Leguminosae 32 espécies. Dentre as 295 espécies, 45 são encontradas na área em estudo.

Foram encontradas 13 espécies consideradas invasoras exclusivas de pastagens, segundo Ferreira \& Laca-Buendia (9) que são assinalados no Quadro 1.

No Quadro 2 são citadas as espécies que aparecem nos quadrados por ordem de Índice de Valor de Importância (IVI). Este indice retrata melhor a comunidade visto que diminui o erro causado pela influência da forma, tamanho e distribuição dos quadrados nos dados de freqüência (7).

Gem tchujnicov et al. (12) afirmam que para a avaliação da abundância de espécies de plantas daninhas ocorrentes em plantações, poucos autores utilizam-se do método exato, isto é, contagem de indivíduos em pequenas parcelas. O método mais comum é a avaliação geral da comunidade e a anotação da ocorrência das espécies na seguinte escala baseada em Maltzew (13) :

U - es pécie encontrada uma única vez no campo todo ;

$\mathrm{R}$ - espécie encontrada raramente $\mathrm{e}$ despercebida no meio da cultura ou forrageira (de 1 a $10 \%$ ) ;

Sol - espécie encontrada em forma de indivíduos solitários, porém de vez em quando aparecendo no meio da cultura de 11 a $20 \%$ ) ;

$\mathrm{Sp}$ - espécie em relativa abundância, porém a cultura dominando (de 21 a $30 \%)$

Cop - espécie distribuída e em alguns lugares prevalecendo sobre a cultura (de 31 a $40 \%$ ).

Utilizando-se este método e o de quadrados chegou-se a resultados semelhantes (Quadro 2) .

As espécies da categoria Cop foram : margaridinha (Wedelia paludosa DC.), capim de-burro (Cynodon dactylon (L.) Pers.) e capim-forquilha (Paspalum conjugatum Berg.). Apesar das duas primeiras espécies aparecerem em apenas três quadrados, apresentaram alto valor de abundância; já o ca pim-forquilha oc or re u em $50^{\circ} \%$ o dos quadrados com uma densidade mais baixa.

Croton urticaefolius Lam. (IVI $=12,59)$, guanxuma (Sidastrum micranthum (St. Hil. ) Fryxell) (IVI $=9,28)$ e vassoura (Sida carpinifolia L.) (IVI $=7,63$ ) com formas de crescimento semelhantes, possuem densidade relativa baixa, embora sejam bem representativas no valor de dominância.

Algumas espécies como por exemplo, mentrasto (Hyptis suaveolens Poit.), enxuga (Vernonia scorpioides (Lam.) Pers.), cordão-defrade (Leonotis nepetaefolia $\mathrm{R}$. Br.), vassoura (Scoparia dulcis L.) e maria-preta (Solanum americanum Mill.) incluidas nas categorias $\mathrm{R}$ e U, apresentam ciclo curto e surgem no pasto em períodos bem limitados.

Dentre as espécies que não apareceram na análise dos quadrados, a maior parte enquadra-se na categoria $U$, sendo que oficial-de-sala (Asclepias curassavica L.), mussambê (Cleome spinosa Jacq.), cipó-cabeludo (Mikania cordifolia Willd.), capim-rabode-gato (Setaria geniculata (Lam.) Beauv.), etc., encontram-se na categoria R.

Trombeta (Datura sp.), lírio-do brejo (Hedychium coronarium Koen.) e lágrima-deNossa Senhora (Coix lacrima-jobi L.), são plantas encontradas em número bem re- 
duzido limitando-se às margens do córrego.

A incidência de plantas consideradas tóxicas ao gado é pequena, restringindose a duas espécies : oficial-de-sala e camará (Lantana camara L.), além de três espécies suspeitas de serem tóxicas : mariapreta, arrebenta-cavalo (Solanum aculeatissimum Jacq.) e fedegoso (Cassia occidentalis L.) (16 ), que apesar de não terem aparecido nos quadrados, algumas podem ser encontradas na pastagem durante todo o ano em pequena quantidade.

O pasto não apresenta uma fisionomia uniforme durante todo o ano, dependendo de fatores tais como : composição e estrutura do solo, pluviosidade e principalmente de regime de pastoreio. Devido ao grau de intensidade com que atuam estes fatores, algumas espécies podem apresentar-se mais ou menos vivazes ; devendo a análise fitossociológica ser realizada em diferentes periodos anuais, para melhor traçar a fisionomia da área.

Guanxuma (Sidastrum micranthum (St. Hil.) Fryxe11), Croton urticaefolius Lam. e vassoura (Sida carpinifolia L.), são espécies persistentes no pasto, apresentando-se bem desenvolvidas quando este é usado pelos bovinos ; sendo invasoras de pastagem, quando não há pastoreio elas são sobrepujadas pelas gramíneas.

Mar garidinha (Wedelia paludosa DC. ) e trapoeraba (Commelina agraria L.) surgem no pasto com maior exuberância nos meses de elevada pluviosidade e temperatura, associada a um pastoreio intenso das gramíneas forrageiras, desenvolvem-se cobrindo parcialmente o pasto. Entretanto, em outras situações, o grau de infestação se reduz, não chegando a causar dano ao valor do pasto.

Todas as gramíneas somadas atin gem 161,18 IVI ; as que formam o cultivo totalizam 114,9 IVI ; as espécies de Gramineae que são biologicamente infestantes podem ser consideradas forrageiras associadas.

\section{AGRADECIMENTOS}

Ao Sr. Dario Junqueira de Andrade por permitir usar as suas terras em nossos estudos.

Aos Docentes e Estagiários da Area de Biologia da Universidade Federal Rural do Rio de Janeiro pelo incentivo.

\section{LTERATURA GITADA}

1. Alechin, W.W. Aims and methods of vegetation ecology. In: Mueller-Dombois, D. \& Ellemberg, H. Plant Community Hypotheses. New York, John Wiley and Sons, 1974. Cap. 3.

2. Almeida, J.R.; Mizuguchi, Y.; Xerez, R. \& Gonçalves, L. Divisāo de recursos alimentares entre populaçōes de Erythraeidae e Ascidae (Acari) e Acaulona brasiliana (Diptera, Tachinidae) que parasitam Dysdercus sp. (Hemiptera, Pyrrhocoridae). In: Reuniâo Anual Sociedade Brasileira para o Progresso da Ciencia, 30., 1979. Resumos.

3. Almeida, J.R.; Mizuguchi, Y.; Cruz, J.L. \& Moraes, G. Polimorfismo melânico e proteção mimética em Dysdercus $s p$. In: Reuniäo Anual Sociedade Brasileira para o Progresso da Ciência, 30.', 1979. Resumos.

4. Araújo, D.D. de \& Peixoto, A.L. Renovação da comunidade vegetal de restinga após uma queimada. In: Congresso Nacional de Botânica, 26:\% Academia Brasileira de Ciências, 1977. 1-17, Anais.

5. Braun-Blanquet, J. Estudios de las comunidades vegetales. Sociologia Vegetal. Buenos Ayres, Acme Agency, 1950. 464 p.

6. Cain, S.A.; Castro, G.M. de O.; Pires, J.M. \& Silva, N.T. da. Application of some phytosociological techniques to Brazilian rain forest. Amer, Journ. Bot. 43:911-941, 1956.

7. Cain, S.A. \& Castro, G.M. de O. Manual of vegetation analysis. New York. Hafner Publishing Company. 1971. 325 p.

8. Curtis, J.T. \& McIntosh, R.P. An upland forest continuum in the prairie-forest border region of Wisconsin. Ecol, 32:476-496. 1951.

9. Ferreira, M.B. \& Laca-Buendia, J.P. del. Espécies consideradas plantas daninhas em áreas cultivadas no Estado de Minas Ge. rais. Planta Daninha. 1(2):16-26. 1978.

10. Gemtchujnicov, I.D.; Almeida, F.M. \& Gomes, P.F. Levantamento fitossociológico de populaçōes de plantas daninhas nos cafezais afetados por Hemileia vastatrix. In: Congresso Nacional de Botdnica, 32. Sociedade Botânica do Brasil, 1972. 205-221, Anais.

11. Gemtchujnicov, I.D. Rudereto-Saccharetum I.D.G., associação fragmentária distinta, 
de ambiente semi-sombreado. In: Congres. so Nacional de Botânica, $25 .^{\circ}$, Sociedade Botânica do Brasil, 1974. Anais: 345-460.

12. Gemtchujnicov, I.D.; Almeida, F.M. \& Lopes, E.A. Análise fitossiológica de pastagens artificiais da Fazenda A.B. (SP) (Nota prévia). In: Congresso Nacional de Botânica, 26. ${ }^{\circ}$ Academia Brasileira de Ciências, 1977. 221-224, Anais.

13. Maltzew, A. 1932. In: Gemtchujnicov, I.D. Rudereto-Saccharetum I.D.G., associaçăo fragmentária distinta, de ambiente semisombreado. In: Congresso Nacional de Botânica, 25.․ Sociedade Botânica do Brasil, 1974. Anais: 345-360.

14. Mizuguchi, Y.; Bragiã̃o, A.; Silva, R.C. \& Almeida, J.R. Coexistência de Dysdercus maurus e $D$. fulvoniger em algodoeiros. In: Reuniāo Anual Sociedade Brasileira para o Progresso da Ciência, 30., 1979. Resumos.

15. Sacco, J. da C. Plantas invasoras dos arrozais. In: Seminario Brasileiro de Herbicidas e Ervas Daninhas, 3.․ Campinas, SP, 1961. 23-46, Anais.

16. Santos, H.L. dos et al. Levantamento de plantas toxicas para bovinos e suspeitas de serem tóxicas no Estado de Minas Ge. rais. EPAMIG, 1-97. 1975.

17. Sarmento, A.C. Invasoras de pastagens em dreas da Estação Experimental de També, Pernambuco. Boletim Técnico do Instituto de Pesquisas Agronómicas, Pernambuco, 3:1-21. 1963,

QUADRO 1 - Listagem das espécies ocorrentes, por ordem alfabética de família.

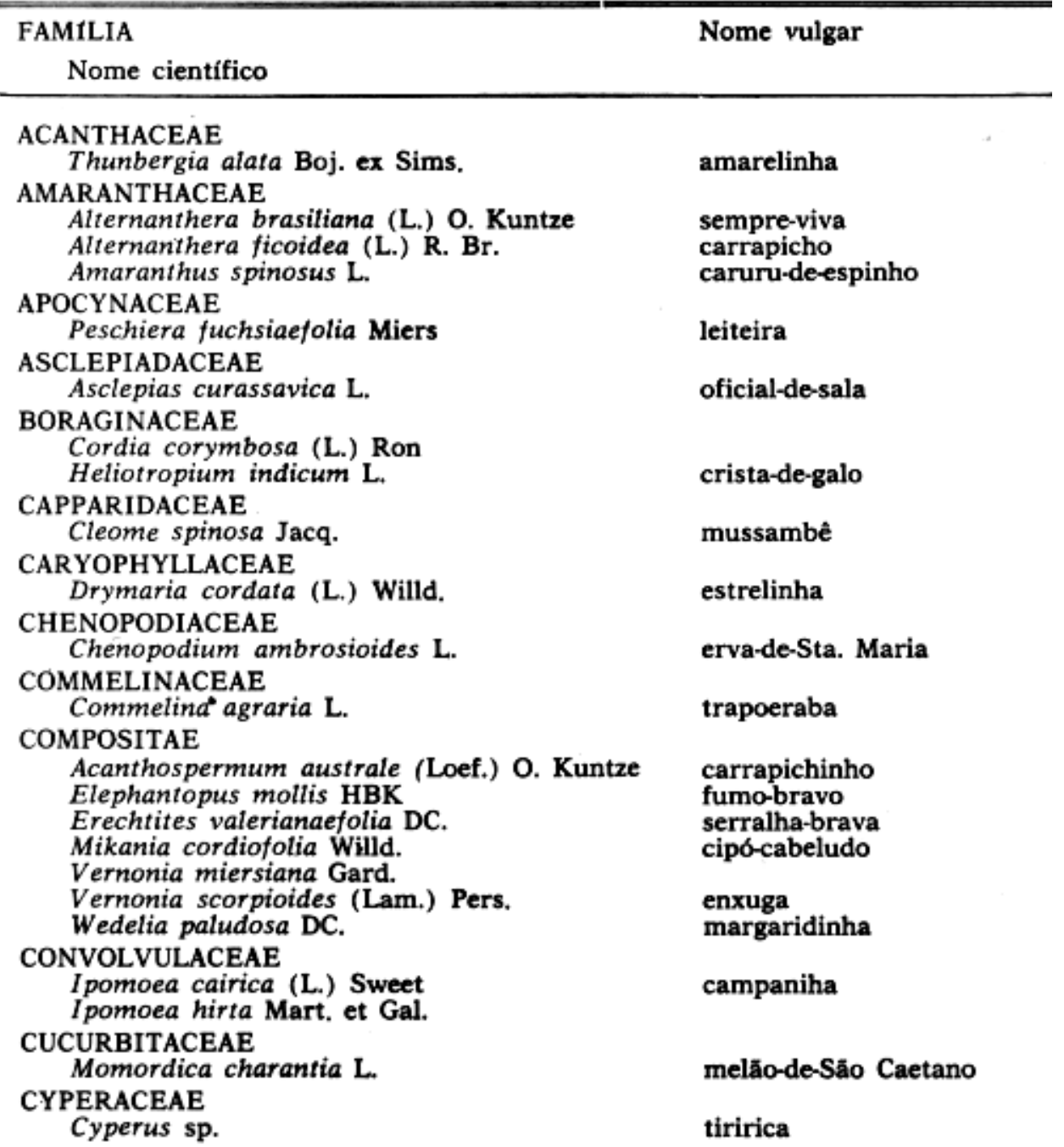


FAMILIA

Nome vulgar

Nome cientifico

EUPHORBIACEAE

Croton urticaefolius Lam.

Euphorbia brasiliensis Lam.

Euphorbia heterophylla $\mathbf{L}$.

Euphorbia pilulifera $\mathbf{L}$.

GRAMINEAE

Brachiaria plantaginea (Link.) Hitch.

Brachiaria purpurascens Henr.

Coix lacrima-jobi $\mathrm{L}$.

Cynodon dactylon (L.) Pers.

Eriochloa polystachya HBK

Paspalum conjugatum Berg.

Paspalum virgatum $\mathrm{L}$.

Pennisetum purpureum Schum.

Setaria geniculata (Lam.) Beauv.

Trichachne insularis (L.) Nees.

LABIATAE

Hyptis lophantha Mart. ex Benht.

Hyptis suaveolens Poit.

Leonorus sibiricus $\mathrm{L}$.

Leonotis nepetaefolia $\mathbf{R}$. Br.

LEGUMINOSAE

Cassia occidentalis $\mathbf{L}$

Cassia tora L.

Centrosema pubescens Benth.

Desmodium canum (Gmel.) Shinz et Thell

Mimosa pudica $\mathrm{L}$.

Phaseolus sp.

Stylosanthes guyanensis (Aubl.) Sw.

LYTHRACEAE

Cuphea mesopotamon Koehne

MALVACEAE

Sida carpinifolia $\mathrm{L}$.

Sida cordifolia L

Sida rhombifolia $\mathrm{L}$.

Sidastrum micranthum (St. Hil.) Fryxell

OXALIDACEAE

Oxalis martiana Zucc.

POLYGONACEAE

Polygonum sp.

RUBIACEAE

Borreria verticillata Mey.

Dioidia rigida Cham et Schlecht

SCHOPHULARIACEAE

Scoparia dulcis $\mathbf{L}$.

SOLANACEAE

Datura sp.

Solanum aculeatissimum Jacq.

Solanum americanum Mill.

Solanum ciliatum Lam.

TILIACEAE

Triumfetta semitriloba Jacq.

VERBENACEAE

Lantana camara $\mathbf{L}$.

Lippia sp.

Stachytarpheta cayennensis (L.C. Rich.) Vahl.

ZIMGIBERACEAE

Hedychium coronarium Koen.

erva-de-Sta. Luzia

leiteira

leiteira

capim-marmelada

capim-angola

lágrmia-de-Nossa Senhora

capim-de-burro

capim-angolinha

capim-forquilha

capim milhä

capim-elefante

capim-rabo-de-gato

capim-amargoso

hortelä

mentrasto

erva-de-S. Francisco

cordão-de-frade

fedegoso

fedegoso

jitirana

carrapicho

dormideira

meladinho

pé-de-pinto

vassoura

malva-branca

vassoura

guanxuma

trevo

vassourinha-branca

vassourinha

vassoura

trombeta

arrebenta-cavalo

maria-preta

açoita-cavalo

carrapicho

camará

gervão

lírio-do-brejo 
QUADRO 2 - Espécies ocorrentes, por ordem do Índice de Valor de Importância (IVI).

\begin{tabular}{|c|c|c|c|c|c|}
\hline Nome científico & $\begin{array}{l}\text { Freqüência } \\
\text { relativa }\end{array}$ & $\begin{array}{l}\text { Densidade } \\
\text { relativa }\end{array}$ & $\begin{array}{l}\text { Dominância } \\
\text { relativa }\end{array}$ & IVI & $\begin{array}{l}\text { Estimativa de } \\
\text { abundância } \mathrm{e} \\
\text { densidade }\end{array}$ \\
\hline Brachiaria plantaginea (Link.) Hitch. & 4,72 & 25,70 & 15,98 & 46,40 & * \\
\hline Pennisetum purpureum Schum. & 4,72 & 24,34 & 11,50 & 40,56 & * \\
\hline Brachiaria purpurascens Henr. & 6,60 & 8,73 & 12,61 & 27,94 & * \\
\hline Wedelia paludosa DC. & 2,83 & 9,13 & 10,69 & 22,65 & Cop \\
\hline Cynodon dactylon (L.) Pers. & 2,83 & 9,13 & 7,23 & 19,19 & Cop \\
\hline Paspalum conjugatum Berg. & 5,66 & 5,72 & 5,17 & 16,55 & Cop \\
\hline Croton urticaefolius Lam. & 4,72 & 1,60 & 6,27 & 12,59 & $\mathrm{Sp}$ \\
\hline Sidastrum micranthum (St. Hil.) Fryxell & 4,72 & 0,65 & 3,91 & 9,28 & Sol \\
\hline Sida carpinifolia $\mathrm{L}$. & 2,83 & 0,70 & 4,20 & 7,73 & Sol \\
\hline Eriochloa polystachya $\mathrm{HBK}$ & 1,88 & 2,76 & 2,50 & 7,14 & Sol \\
\hline Cordia corymbosa (L.) Ron & 2,83 & 0,25 & 3,76 & 6,84 & Sol \\
\hline Sida rhombifolia $\mathrm{L}$. & 4,72 & 0,40 & 1,42 & 6,54 & Sol \\
\hline Cyperus sp. & 0,94 & 2,90 & 2,14 & 5,98 & Sol \\
\hline Polygonum sp. & 3,77 & 0,85 & 0,71 & 5,33 & Sol \\
\hline Solanum aculeatissimum Jacq. & 1,88 & 0,10 & 2,56 & 4,54 & $\mathrm{R}$ \\
\hline Desmodium canum (Gmel.) Schinz et Thell & 2,83 & 0,80 & 0,71 & 4,34 & $\mathrm{R}$ \\
\hline Commelina agraria $\mathrm{L}$. & 2,83 & 1,05 & 0,42 & 4,30 & $\mathrm{R}$ \\
\hline Thunbergia alata Boj. ex Sims & 2,83 & 0,50 & 0,50 & $\begin{array}{l}4,50 \\
3,83\end{array}$ & $\mathrm{R}$ \\
\hline Elephantopus mollis HBK & 2,83 & 0,50 & 0,47 & $\begin{array}{l}5,03 \\
3,80\end{array}$ & $\mathrm{R}$ \\
\hline Drymari acordata (L.) Willd. & 2,83 & 0,75 & 0,07 & 3,65 & $\mathrm{R}$ \\
\hline Paspalum virgatum $\mathbf{L}$. & 2,83 & 0,35 & 0,22 & 3,40 & $\mathbf{R}$ \\
\hline Mimosa pudica $\mathrm{L}$. & 1,88 & 0,60 & 0,81 & 3,29 & $\mathbf{R}$ \\
\hline Stachytarpheta cayennensis (L.C. Rich.) Vahl. & 2,83 & 0,20 & 0,25 & 3,28 & $\mathbf{R}$ \\
\hline Vernonia scorpioides (Lam.) Pers. & 1,88 & 0,15 & 1,14 & 3,17 & $\mathbf{R}$ \\
\hline Hyptis suaveolens Poit. & 1,88 & 0,15 & 0,96 & 2,99 & $\mathbf{R}$ \\
\hline Ipomoea cairica (L.) Sweet & 1,88 & 0,55 & 0,35 & 2,78 & $\mathbf{R}$ \\
\hline Alternanthera ficoidea (L.) $\mathrm{R} . \mathrm{Br}$. & 1,88 & 0,15 & 0,71 & 2,74 & $\mathbf{R}$ \\
\hline Centrosema pubescens Benth. & 1,88 & 0,15 & 0,50 & 2,53 & $\mathbf{R}$ \\
\hline Acanthospermum australe (Loef.) O. Kuntze & 1,88 & 0,15 & 0,35 & 2,38 & $\mathbf{R}$ \\
\hline Oxalis martiana Zucc. & 1,88 & 0,25 & 0,11 & $\begin{array}{l}2,30 \\
2,24\end{array}$ & R \\
\hline Phaseolus sp. & 1,88 & 0,20 & 0,11 & 2,19 & $\mathbf{R}$ \\
\hline Euphorbia heterophylla L. & 1,88 & 0,15 & 0,11 & $\begin{array}{l}2,19 \\
2,14\end{array}$ & $\mathbf{R}$ \\
\hline Dioidia rigida Cham et Schlecht & 1,88 & 0,15 & 0,05 & $\begin{array}{l}2,14 \\
2,08\end{array}$ & $\mathbf{R}$ \\
\hline Chenopoduim ambrosioides $\mathrm{L}$. & 0,94 & 0,10 & 0,71 & $\begin{array}{l}2,00 \\
1,75\end{array}$ & $\mathbf{R}$ \\
\hline Solanum ciliatum Lam. & 0,94 & 0,15 & 0,57 & 1,66 & $\mathbf{R}$ \\
\hline Vernonia miersiana Gard. & 0,94 & 0,15 & 0,32 & 1,41 & $\mathbf{R}$ \\
\hline Leonotis nepetaefolia $\mathrm{R}$. Br. & 0,94 & 0,10 & 0,28 & 1,32 & $\mathbf{R}$ \\
\hline
\end{tabular}

Obs..: * cultivada 